\title{
Two-step retrograde closed stenting: a novel method for treating canalicular lacerations in Chinese patients
}

Y Bi ${ }^{1,2,4,5}$, G Sui ${ }^{3,4,5}$, Q Zhou ${ }^{1,4,5}$, LM Heindl ${ }^{2}$,

F Bock ${ }^{2}$, X Sun ${ }^{1}$, S Tang $^{1}$, Z W Wang ${ }^{1}$ and

C Cursiefen ${ }^{2}$

\begin{abstract}
Purpose To evaluate the efficacy of two-step retrograde closed stenting for treating canalicular laceration.

Methods Forty-eight consecutive canalicular laceration cases (48 eyes) were randomised and divided into two groups: a one-step group and a two-step group. In the two-step group ( 23 cases), the first step was performed in the outpatient department and included identifying the medial cut end of the canaliculus and probing under a slit-lamp microscope, followed by a retrograde canalicular stenting assisted by a memory titanium stylet. The second step was canalicular anastomosis, which was performed in the operating room. In the one-step group (25 cases), all of the surgical procedures were performed when preoperative preparations were simultaneously available.

Results The time elapsed from the doctor visit to the treatment was $4.3 \pm 2.4 \mathrm{~h}$ in the two-step group and $18.8 \pm 6.3 \mathrm{~h}$ in the onestep group $(P<0.01)$. The canalicular medial cut ends were found in all cases, but $8.6 \pm 3.5 \mathrm{~min}$ was needed in the two-step group, and $51.4 \pm 24.2 \mathrm{~min}$ was needed in the one-step group $(P<0.01)$. The numerical rating scale for pain during surgery was $1.8 \pm 1.2$ in the two-step group and $5.4 \pm 2.2$ in the one-step group $(P<0.01)$. One case $\mathbf{2} .63 \%)$ in the two-step group and nine cases $(36 \%)$ in the one-step group required other assisted methods to locate the medial cut end $(P=0.007)$. Twenty-one cases $(91.3 \%)$ in the two-step group and 20 cases $(80 \%)$ in the one-step group achieved patent lacrimal drainage systems during a 12-month follow-up $(P=0.528)$.
\end{abstract}

Conclusions The two-step canalicular anastomosis method allows an early search for the medial cut end of the canaliculus and improves the chances of finding it; it is also a quicker, less invasive method for treating canalicular lacerations.

Eye (2013) 27, 1275-1280; doi:10.1038/eye.2013.169; published online 23 August 2013

Keywords: canalicular laceration; canalicular stenting; canalicular anastomosis; lacrimal surgery

\section{Introduction}

Canalicular lacerations have been reported in all age groups and are a usual finding in ophthalmic emergencies, frequently affecting the lower canaliculus. ${ }^{1}$ The primary management goal in these cases is to restore a patent canalicular system. This goal is achieved by meticulous anastomosis and temporary stenting of the separated canalicular cut ends. ${ }^{2}$ Another goal is ensuring that the lacrimal punctum is returned to its previous physiological position without secondary eversion or slitting. ${ }^{3}$ In situ canalicular anastomosis is also an important precondition for acquiring a normal eyelid position. ${ }^{4}$ Although canalicular laceration management varies widely, it is generally agreed that an immediate anastomosis and stenting is necessary; the medial cut end may retreat and can be difficult to identify $24-48 \mathrm{~h}$ after injury because fibrin and granulation deposition occurs quickly. ${ }^{5,6}$ Usually, a long period of time elapses between the trauma and the doctor visit, and the preoperative preparation is time consuming; to a large degree, these delays can
${ }^{1}$ Department of Ophthalmology, Tongji Hospital affiliated with Tongji University School of Medicine, Shanghai, China

${ }^{2}$ Department of Ophthalmology, University Hospital of Cologne, Cologne, Germany

${ }^{3}$ Department of Ophthalmology, Jilin University Bethune Second Hospital, Changchun, China

Correspondence:

C Cursiefen, Department of Ophthalmology, University Hospital of Cologne,

Kerpener Straße 62, 50937, Cologne, Germany. Tel: + 49221478 4300; Fax: + 492214785094 E-mail: ccursiefen@yahoo. com

${ }^{4}$ These authors contributed equally to this work.

${ }^{5}$ Equivalent first authors.

Received: 8 April 2013 Accepted in revised form: 13 June 2013 Published online: 23 August 2013 
hinder immediate anastomosis after canalicular lacerations. Searching for the medial cut ends is usually the most difficult part of the surgery, and because this procedure can waste time, it requires an experienced physician. The medical resource distribution is imbalanced between cities and even between university hospitals; therefore, it is unreasonable to ask experienced doctors specialising in the lacrimal system to wait for several hours or even days until the operating room, nurse, and surgeons are simultaneously available. In this study, we developed an optimised surgical procedure that facilitated finding the medial cut end of the lacerated canaliculus earlier.

\section{Materials and methods}

\section{Patients}

Between June 2009 and February 2011, 48 patients (48 eyes) suffering from monocanalicular lacerations at the Tongji University Hospital were prospectively followed up. The following traumas were observed in this patient group: blunt trauma (35 cases), including fist or finger contusions $(41.7 \%)$, tree branch or protruding objects scratches (20.8\%), and throwing objects injuries (10.4\%); and sharp injuries (13 cases), including being cut by glass $(18.8 \%)$ and being cut by a knife $(8.3 \%)$. The canalicular laceration diagnosis was based on an ophthalmic examination, lacrimal irrigation, and probing. Based on computer-generated randomised numbers, the patients were separated into two groups. In the two-step group (23 cases), the canaliculus medial cut end was directly identified under a slit-lamp microscope and was followed by an immediate retrograde stenting in the outpatient department (first step). Then, the separated canaliculus cut ends were later anastomosed under a surgical microscope until the operating room became available (second step). In the one-step group (25 cases), the patients waited several hours or days until the operating room, nurse, and doctor were available at the same time, and then all the procedures were performed together under a surgical microscope.

Before surgery, all of the patients received topical anaesthetic eye drops (proparacaine hydrochloride) and an anaesthetic solution ( $2 \%$ lidocaine with epinephrine) local injection. The injections included $2 \mathrm{cc}$ in the infratrochlear region, $1 \mathrm{cc}$ in the infraorbital region, and $1 \mathrm{cc}$ inside the lacrimal sac. The following data were collected from each patient: the age, gender, affected location, causes of trauma, time from trauma to doctor visit, time from doctor visit to surgical intervention, extent of canalicular and eyelid injury, time spent in searching for the medial cut end, and stenting duration (Table 1). The search for the canalicular medial cut ends and the retrograde stenting were performed by either of

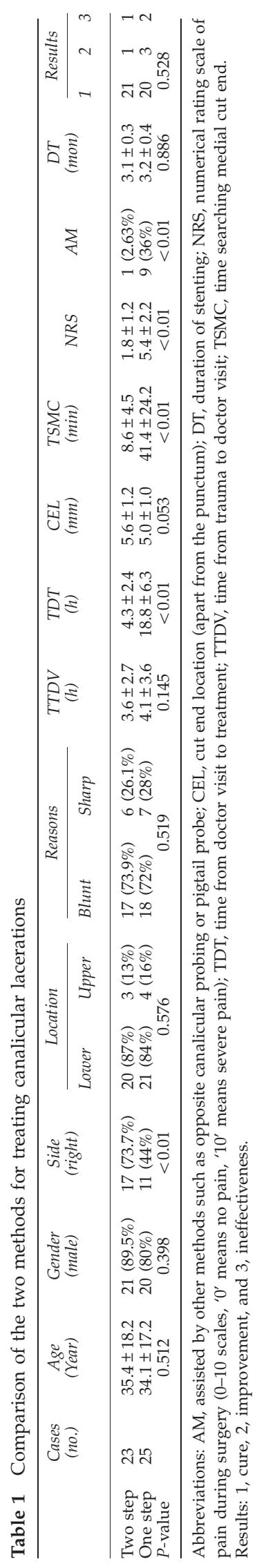


the two experienced surgeons (Yanlong Bi or Guiqin Sui). Canalicular anastomosis was usually performed by other surgeons. The following exclusion criteria were applied: patients who were uncooperative during surgery (eg, being intoxicated), did not agree to stenting, presented with both upper and lower canalicular lacerations, presented with uncontrollable bleeding, the time from the trauma to the doctor visit exceeded $24 \mathrm{~h}$, coexisting intraocular injuries, or nasal bone fractures.

\section{Surgical procedures}

All surgical procedures were performed under local anaesthesia and abided by the basic aseptic principle. The study was approved by the local ethics committee and carried out in conformance with the tenets of the Helsinki Declaration.

The first step of the two-step stenting method was performed in the outpatient department. Sufficient haemostasis usually could be achieved with wound compression for several minutes after local anaesthesia. To fully expose the injury field under the slit-lamp microscope, the patient's head was tilted $20^{\circ}-35^{\circ}$ towards the injured side. A sterile tubular probe (20G with $0.58 \mathrm{~mm}$ inner diameter and $0.9 \mathrm{~mm}$ outer diameter) (Figures 1a-d) containing a memory titanium guiding stylet was held in the surgeon's right hand for the left injury side and in the left hand for the right injury side. The surgeon took a sterile cotton swab with the free hand
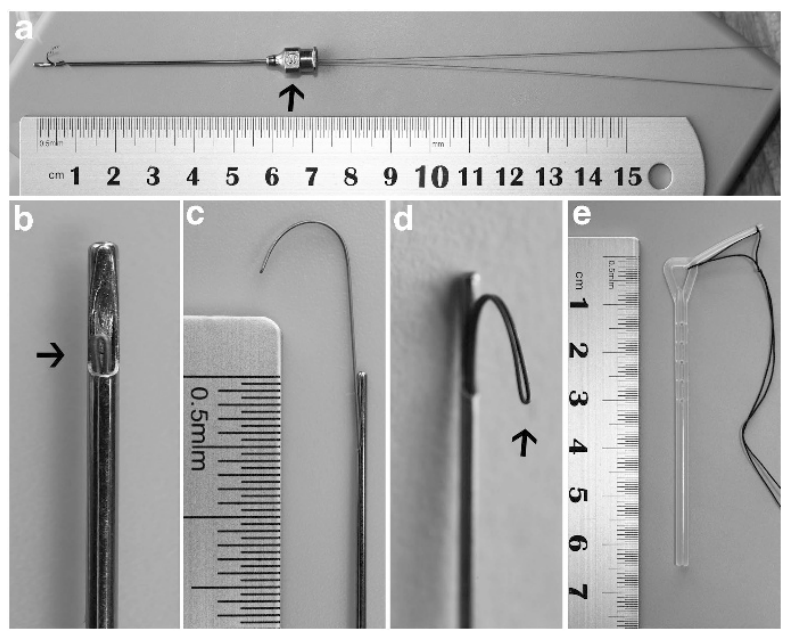

Figure 1 (a) The 20G tubular probe, which contained a doublestrand memory titanium guiding stylet. Letter ' 9 ' (arrow) helped to locate the direction of the stylet. (b) Probe opening and stylet hidden inside (arrow). (c) The memory titanium returning to a curved shape after pushing out of the probe and (d) a ring tip could be observed (arrow), which helped to catch the suture binding on a stent. (e) The ' $Y$ ' shape silicon stent with a lateral canalicular tube. to assist in exposing the traumatic field and drying the blood (Figure 2a). The medial cut end of the canaliculus was easily exposed and typically had a rolled white edge and a shiny epithelial lining. Occasionally, when the medial cut end was difficult to identify, opposite canalicular probing or pigtail probe would be adopted. ${ }^{7}$ The 20G probe was antegrade inserted until the tip of the probe reached the inferior nasal meatus. The patient then maintained a supine position, and nasal packing was performed. The packing set included several cotton strips, which were previously soaked in ephedrine and Furacilin nasal drops and proparacaine hydrochloride solution. Five minutes later, the nasal packing was removed, and the probe's direction was adjusted. The memory stylet was pushed downward and emerged from the nostril after bypassing the inferior turbinate. This process was primarily dependent upon the stylet's memory property, which helped the recovery to its initial curved shape (Figures $2 b$ and $3 a$ ). A silicon stenting integrated with a lateral canaliculus tube was retrograde retracted from the inferior meatus into the lacrimal duct (Figure 1e), and at that time, the lateral canaliculus tube with the binding sutures extending from the medial cut end could be identified in the injury field (Figure 2c). a
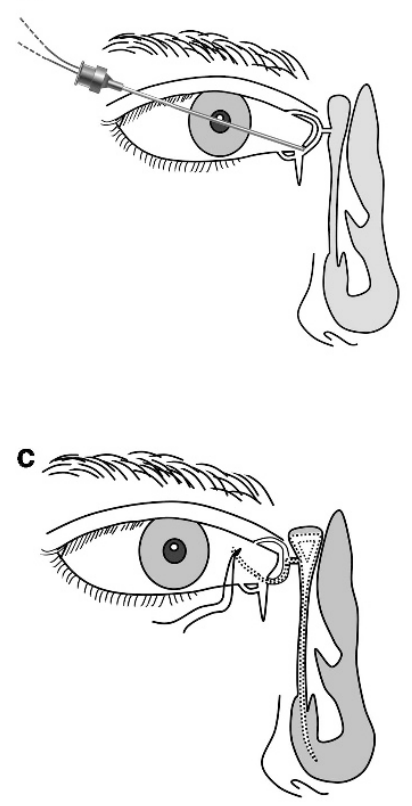

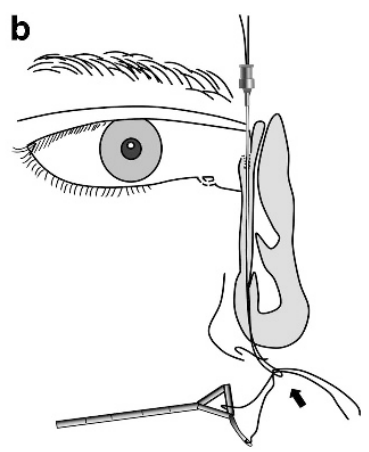

d

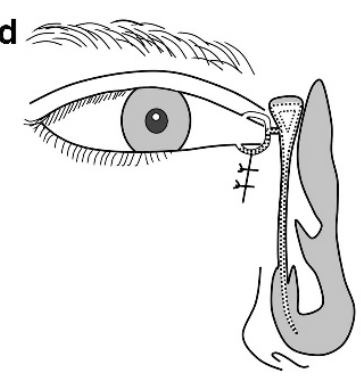

Figure 2 (a) Canalicular medial cut end searching and probing under a slit-lamp microscope. (b) After antegrade probing, the stylet was pushed out of the nostril, catching the sutures binding on the stent (arrow). (c) The lateral canaliculus tube and the binding sutures extended from the medial cut end and could be found in the injury field. (d) The stent was stuck inside the lacrimal duct, including canalicular stenting. 

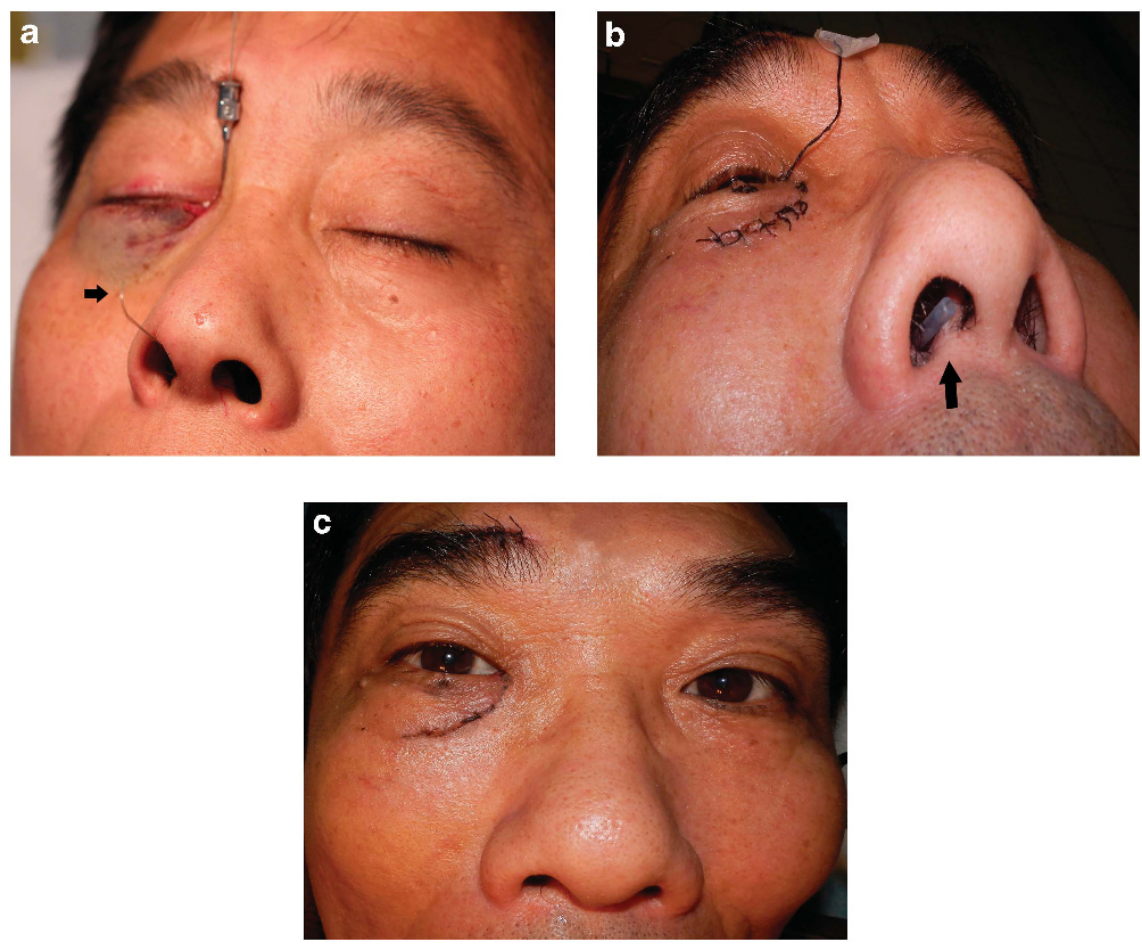

Figure 3 A patient with right lower canalicular laceration treated by the two-step retrograde closed stenting. (a) The memory stylet extended from the nostril with no intra nostril assistance (arrow). (b) Within 1-week post-surgery, the redundant lateral canalicular tube and sutures were not trimmed. The stent could be observed when looking upward (arrow). (c) One-week post-surgery, the stent was invisible in a normal head position after the sutures were removed.

In the second step, under the surgical microscope, the lateral canaliculus tube and the binding sutures were guided, passing the temporal canaliculus cut end, and pulled out of the punctum. For the canaliculus anastomosis, the twisted, separated canaliculus was stretched along the lateral canaliculus tube, and three interrupted $8 / 0$ vicryl sutures were meticulously passed though not only the canalicular walls but also the submucosa. The dissociative and/or everted eyelids could usually acquire an anatomical reposition after the sutures were tied. The deep tissue and skin were closed thereafter (Figure 2d).

In the one-step stenting method, following several hours of waiting and after local anaesthesia was administered, the procedures were performed simultaneously under the surgical microscope in the operating room.

\section{Postoperative care}

The pain scale assessment score ( $0-10$ scale, ' 0 ' means no pain, ' 10 ' means severe pain) was recorded the second day post-surgery (Table 1). The patients were evaluated at 1 week, 1 month, and 3 months, and every 2 months thereafter until 1-year post-surgery. Postoperatively, all patients were treated with antibiotic eye drops four times daily and instructed to be careful when wiping or kneading the nose. One-week post-surgery, after the swelling subsided, the exposed binding canaliculus suture and the redundant lateral canaliculus tube were trimmed (Figures $3 b$ and c). Three months post-surgery, the silicon tube was removed from the nostril by gently turning and pulling with two vessel forceps.

\section{Criteria defining the clinical effects}

The clinical effects were classified in three levels, which were confirmed by probing and lacrimal irrigation:

(1) cure (no epiphora), with complete patent canaliculus; (2) improvement (slight or no epiphora), with canalicular partly stenosis; and (3) ineffectiveness, complete canalicular obstruction.

\section{Statistical analysis}

Data collection and statistical analyses were performed using Microsoft Office EXCEL 2010 and SPSS (Statistical Package for the Social Sciences) Version 13.0 (SPSS, Inc., Chicago, IL, USA). Chi-square tests and $t$-tests were used to determine the statistical significance. A difference was considered significant when $P<0.05$. 


\section{Results}

\section{Clinical effects}

The baseline data, surgical parameters, and postoperative results are shown in Table 1 . The silicone stents were left in place for at least 3 months in most cases, except for two cases when the individuals pulled the stents from their nostrils by themselves (one within 1 month and one within 2 months post-surgery). No premature stent loss occurred accidentally. The mean follow-up period was 11-16 months (mean $13.1 \pm 3.7$ months) in the two-step group and 11-15 months (mean 12.8 \pm 3.6 months) in the one-step group. A patent canaliculus could be achieved in $91.3 \%$ of patients in the two-step group and $80 \%$ of patients in the one-step group $(P=0.528)$.

\section{Complications}

None of the patients in either group complained of epiphora, including the patients with canalicular stenosis or obstruction. The most frequent complaint of nearly all of the patients $(96.2 \%)$ was a varying degree of swelling sensation beginning shortly after stenting. However, this complaint gradually became less obvious 2 weeks after the procedure. There were no complications, such as granuloma, fistula, dacryocystitis, or punctal slitting. None of the patients reported that the stent could be perceived during social communication.

\section{Conclusion}

For lacerated canaliculus anastomosis, the initial step is to locate the canalicular medial cut end. This procedure is traditionally followed with canalicular stenting; both procedures are performed concurrently in the operating room, where surgical registration and preparation by the nurses are needed. ${ }^{1-5}$ The patients always waited for several hours, or even days, until a nurse, the operating room, and the experienced ophthalmologist were simultaneously available. Experienced ophthalmologists at a large hospital often treat more than one patient at a time, and it is particularly illogical to always ask them to wait for the uncertain emergent surgical schedule during their rest time. Herzum et $a l^{7}$ reported that the average waiting time from canalicular injury to surgery was $3.3 \mathrm{~h}$; in another report, a $32 \mathrm{~h}$ waiting period was observed. ${ }^{8}$ As time elapses, the canalicular medial cut end gradually becomes more difficult to identify. ${ }^{5}$ We reviewed the clinical and histopathologic data from 350 consecutive patients who underwent primary dacryocystorhinostomy and observed that in late surgery, scarred contracted tissues of the lacrimal passage could generate difficulties in creating an anastomosis, and this issue confirmed that early surgery should be undertaken. ${ }^{6}$
In our study, we separated the procedure of identifying the lacerated canalicular medial cut end from the whole canalicular anastomosis surgery by performing this procedure under the slit-lamp microscope in the outpatient department. This optimisation dramatically shortened the time elapsed from the doctor visit to the surgical intervention (from $18.8 \pm 6.3 \mathrm{~h}$ in the one-step group to $4.3 \pm 2.4 \mathrm{~h}$ in the two-step group, $P<0.01$ ), shortened the time used in searching for the medial cut end (from $41.4 \pm 24.2 \mathrm{~min}$ in the one-step group to $8.6 \pm 4.5 \mathrm{~min}$ in the two-step group, $P<0.01$ ), and decreased the failure rate of the direct visualisation medial cut end search (from $36 \%$ in the one-step group to $2.63 \%$ in the two-step group, $P=0.007$ ). Correspondingly, this optimisation relieved the patients' pain, which was confirmed by the NRS (5.4 \pm 2.2 in the one-step group and $1.8 \pm 1.2$ in the two-step group, $P<0.01$; Table 1 ).

For the canalicular stenting, one essentially must choose from either a bicanalicular nasal stenting, an annular stent assisted by pigtail probe, or a monocanalicular stenting. ${ }^{1-4}$ When evaluated for invasiveness, the nasal stenting is traditionally considered a more invasive technique because it is usually difficult to clamp the inserted antegrade suture crossing the inferior turbinate out of the inferior meatus, and it often needs to be assisted by an intranasal endoscope. ${ }^{9,10}$ Sometimes, this process is time consuming. In all of our 78 cases, the memory titanium guiding stylet could be easily self-retrieved out of the nostril without the assistance of additional instruments, and no patient experienced iatrogenic bleeding or other complications during the retrieval process. The mean time used for the stylet retrieval was $3.6 \pm 2.4 \mathrm{~min}$. In our experience, this process was much quicker compared with the traditional intranasal clamping method. Two points should be emphasised: (1) an adequate presurgical nasal packing is necessary to shrink the inferior turbinate and the surrounding mucosal tissue, which could facilitate the retrieval to a greater extent; and (2) because individual anatomical differences exist, the stylet may astray in the throat; if it does, the surgeon should withdraw the stylet and adjust the probe's direction or deepness. In our experience, within 3-5 times of moving the stylet back and forth, the tip of the stylet could emerge from the nostril.

In this study, the ' $Y$ ' shaped silicon stent was with a lateral canalicular tube. The stent has a certain elasticity and tension, and after retrogradely placing it into the lacrimal duct, the tip (' $\mathrm{V}$ ' shaping) could become stuck inside the lacrimal sac. No stent displacement was found in our patients. After canalicular anastomosis, the lateral canalicular tube was placed exactly inside the canaliculus. The excessive lateral tube should be trimmed at least 1 week after surgery to avoid 
inappropriate punctum-tube positioning after eyelid oedema subsides. Unlike with monocanalicular and bicanalicular stenting, this retrograde stent was absolutely hidden inside the lacrimal duct with no visible tube or sutures. This result could be the reason why complications, such as punctual slitting, punctal granuloma formation, and corneal erosions, were not found in our patients. ${ }^{11,12}$ The stent could not be perceived during social communication; to a large extent, this feature helped maintain good compliance $(95.8 \%)$ in our patients. The main patient complaint was a nasal swelling sensation within 2 weeks post-surgery, but this sensation could be tolerated by most patients.

In this study, we advocated a novel two-step procedure to optimise the canalicular anastomosis. We also introduced the use of a memory titanium guiding stylet, which simplified the retrograde stenting process, and a closed retrograde canalicular stenting for the lacerated canaliculus. Although the clinical effects, as judged by the lacrimal irrigation system, did not differ between the two groups $(P=0.528)$, compared with the traditional method, the two-step canalicular laceration repairing method was less invasiveness, offered a shorter operating time, and was a simpler procedure. What we value most is that this two-step method is also a reasonable medical resource allocation method that could adequately improve the working efficiency of a senior ophthalmologist. It is suggested that two-step retrograde closed stenting should be considered as an alternative method in treating canalicular lacerations.

\section{Summary}

What was known before

- The steps of canalicular anastomosis are performed simultaneously.

- The patients always wait several hours or even days until medical intervention.

- Few experienced ophthalmologists are specialised in the lacrimal system, and they are not equally distributed in China.

What this study adds

- We introduced a less invasive two-step canalicular laceration repair method, which allows an early search for the medial cut end and improves the chances of finding it.

- The two-step canaliculus repairing method helps to reasonably improve the working efficiency of senior ophthalmologists.

- Retrograde closed stenting using a Y-shaped silicon stent is an alternative choice for repairing the canalicular laceration.

\section{Conflict of interest}

The authors declare no conflict of interest.

\section{Acknowledgements}

Funding for this study comes from the Natural Science Foundation of China (NSFC: 30973247) and the Shanghai Excellent University Teacher Foundation (1500144019).

\section{References}

1 Jordan DR, Mawn L. Repair of canalicular lacerations. Am J Ophthalmol 2008; 146(5): 792-793.

2 Naik MN, Kelapure A, Rath S, Honavar SG. Management of canalicular lacerations: epidemiological aspects and experience with Mini-Monoka monocanalicular stent. Am J Ophthalmol 2008; 145(2): 375-380.

3 Komínek P, Cervenka S, Pniak T, Zeleník K, Tomášková H, Matoušek P. Monocanalicular versus bicanalicular intubation in the treatment of congenital nasolacrimal duct obstruction. Graefe's Arch Clin Exp Ophthalmol 2011; 249(11): 1729-1733.

4 Tint NL, Alexander P, Cook AE, Leatherbarrow B. Eyelid avulsion repair with bi-canalicular silicone stenting without medial canthal tendon reconstruction. Br J Ophthalmol 2011; 95(10): 1389-1392.

5 Lindsey JT. Lacrimal duct injuries revisited: a retrospective review of six patients. Ann Plast Surg 2000; 44(2): 167-172.

6 Heindl LM, Junemann A, Holbach LM. A clinicopathologic study of nasal mucosa in 350 patients with external dacryocystorhinostomy. Orbit 2009; 28(1): 7-11.

7 Herzum H, Holle P, Hintschich C. Eyelid injuries: epidemiological aspects. Ophthalmologe 2001; 98(11): 1079-1082.

8 Naik MN, Kelapure A, Rath S, Honavar SG. Management of canalicular lacerations: epidemiological aspects and experience with Mini-Monoka monocanalicular stent. Am J Ophthalmol 2008; 145(2): 375-380.

9 Wu SY, Ma L, Chen RJ, Tsai YJ, Chu YC. Analysis of bicanalicular nasal intubation in the repair of canalicular lacerations. Jpn J Ophthalmol 2010; 54(1): 24-31.

10 Chen D, Li N, Wan P, Xiao J, Liu Y, Wang X et al. A novel procedure for treating canalicular obstruction by re-canaliculisation and bicanalicular intubation. Br J Ophthalmol 2012; 96(3): 366-369.

11 Shah A, Tekriwal AK, Drummond PM, Woodruff G. Long-term results of closed nasolacrimal intubation in adults. Eur J Ophthalmol 2007; 17(4): 490-493.

12 Madge SN, Selva D. ANZSOPS DCR Intubation Study Group. Canalicular intubation in routine dacryocystorhinostomy. Clin Experiment Ophthalmol 2009; 37(5): 533-534. 